\author{
Beat Meier \\ Department of Psychology and Center for Cognition, Learning and Memory \\ University of Bern, Switzerland \\ Address correspondence to: \\ Beat Meier \\ Department of Psychology \\ University of Bern \\ 3000 Bern 9, Switzerland \\ e-mail: beat.meier@psy.unibe.ch \\ Phone: +41316314039 \\ Fax: + 41316318212
}

\title{
Semantic representation of synaesthesia
}

\begin{abstract}
Synaesthesia has multifaceted consequences for both subjective experience and cognitive performance. Here, I broach the issue of how synaesthesia is represented in semantic memory. I hypothesize that, for example, in grapheme colour synaesthesia, colour is represented as an additional feature in the semantic network that enables the formation of associations that are not present in nonsynaesthetes. Thus, synaesthesia provokes richer memory representations which enable learning opportunities that are not present in non-synaesthetes, provides additional memory cues, and may trigger creative ideas.
\end{abstract}

Keywords: synaesthesia; semantic representation system; semantic memory; unidirectionality; bidirectionality; startle response; cognitive style.

In synaesthesia ordinary stimuli trigger untypical experiences. For example, the letter A printed in black may also trigger a particular colour experience (e.g., red). The experience is consistent, triggered automatically, and typically has always been there, since a synaesthete can remember (cf. Baron-Cohen et al. 1987; Grossenbacher and Lovelace 2001). About two to five per cent 
of the general population are affected by this condition (Simner et al. 2006). Most typical are inducers such as digits, letters, words, days of the week, months, or sound and the concurrent experience is most typically related to colour or space (Novich et al. 2011). However, many more combinations are possible (see the list of synaesthesias by Sean Day; 2013). It has been established as a convention that the particular form is termed according to its inducer-concurrent pairs (e.g., grapheme-colour). As most research has been conducted on grapheme-colour synaesthesia, here I focus on this particular form, although some of the remarks are more general, and although it must be noted that many synaesthetes have more than one form of synaesthesia.

Recent research has made progress in showing that the condition is real; that is, it has a neurological basis. There is converging evidence from functional imaging studies, diffusion tensor imaging, and voxelbased morphometry that temporal regions related to processing form and colour and parietal areas related to binding processes are critically involved in (grapheme-colour) synaesthesia (Hubbard et al. 2005; Hubbard, Brang, and Ramachandran 2011; Rouw and Scholte 2007, 2010; Weiss and Fink 2009). Moreover, findings based on event-related potentials suggest that synaesthetes may differ in magno- and parvo-cellular processing (Barnett et al. 2008). In addition, a recent study has suggested that synaesthesia is accompanied by globally altered brain network topology (Hänggi et al. 2011).

Moreover, synaesthesia can have consequences for cognitive performance, in particular for memory (e.g., Yaro and Ward 2007; Rothen and Meier 2010a; Radvansky, Gibson and McNerney 2011; see Rothen, Meier, and Ward 2012; Meier and Rothen 2013, for recent reviews). Thus, synaesthesia may provide additional retrieval cues which can be used to retrieve information from memory. However, as there is also some evidence that synaesthetes have a memory advantage even for materials that do not trigger synaesthesia (e.g., Rothen and Meier 2010a), this seems not to be the whole explanation. It is possible that synaesthetes may have a generally enriched world of experience and that this is directly related to the available semantic representations. I will elaborate on this point below.

Originally, it has been thought that synaesthesia can be characterized as a perceptual phenomenon (Baron-Cohen and Harrison 1997; Laeng et al. 2004; Mattingley et al. 2001; Palmeri et al. 2002; Ramachandran and Hubbard 2001a). For example, Ramachandran and Hubbard (2001a) used a visual search task to investigate whether synaesthetic experiences are genuinely perceptual. They presented different geometric shapes consisting of the digits that triggered particular colour experiences for two synaesthetes. 
The result showed that the synaesthetes recognized more shapes than a control group. Moreover, in another experiment, presenting a flanked grapheme in the periphery of the visual field evoked colour even though the grapheme itself could not be identified. These results suggest that synaesthesia occurs at an early stage of perceptual processing. However, follow-up studies using many different variants of the visual search task, did not replicate this result (Edquistet al. 2006; Rothen and Meier 2009; Sagiv et al. 2006; Ward et al. 2010). These discrepant results can be explained by individual differences between synaesthetes (e.g., lower vs. higher). However, in addition, the latter results at least seem to suggest that synaesthesia is not purely a perceptual condition.

In line with these considerations, research using ambiguous graphemes, for example where a 2 can be perceived as a $\mathrm{Z}$ or a 5 as an $\mathrm{S}$, showed that, depending on the context, the same physical stimulus can be perceived in a different colour, depending on the context-specific interpretation (Myles et al. 2003; Dixon et al. 2006). Moreover, when an arithmetic problem is presented (e.g., $5+2$ ) and immediately afterwards the colour of a colour patch must be named, naming times are faster when the synaesthetic colour of the solution to the calculation is congruent to the colour patch (Dixon et al. 2000). This result suggests that activating the conceptual representation of an inducer is sufficient to trigger a synaesthetic response. In fact, synaesthetic experiences can even occur for imagined synaesthetic inducers (Frith and Paulesu 1997; Ramachandran and Hubbard 2001b).

Further support for the presence of a strong conceptual contribution to synaesthesia comes from research on implicit bidirectionality. Although at the level of subjective experience the activation of the concurrent (e.g., colour) is typically unidirectional - from the inducer to the concurrent, performance on an inducer stimulus may also be biased by an implicit activation of a concurrent. For example, reversing the usual synaesthetic Stroop test, Johnson et al. (2007) found that responding to incongruentlycoloured stimuli was slowed compared to congruently coloured stimuli. Brugger et al. (2004) found faster left-hand responses for colours associated with small numbers and faster right-hand responses for colours associated with large numbers in digit-colour synaesthetes. Cohen Kadosh and Henik (2006) asked digit-colour synaesthetes to decide which one of two digits was numerically larger. They found a facilitation of performance whenever the synaesthetic colours elicited by the digits indicated a larger distance than the relevant numerical values. Knoch et al. (2005) used a modified random generation task in which they asked digit-colour synaesthetes to generate random sequences of colours. Their results showed a similar 
pattern of counting bias as it is known from traditional number generation tasks. Weiss et al. (2009) presented participants with a word-completion task in which letter strings could be turned into a high- or low-frequency word by adding a different first-letter. When the space for the first letter was primed by the colour of the letter that would form the low-frequency word, performance of the synaesthetes was in fact biased towards completing the low-frequency word.

Moreover, with a psycho-physiological method, we have shown that a startle response that is conditioned to a particular colour is also elicited when a grapheme is presented that induces that particular colour (Meier and Rothen 2007). As the synaesthetic inducer never co-occurred with the startling sound, we have argued that an indirect activation of the grapheme representation (i.e., through implicit bidirectionality) was responsible for the establishment of an association with the startle response. This association was re-activated and fired back when the grapheme was presented physically, which resulted in another conditioned startle response. In a follow-up study, we have demonstrated that, by suppressing parieto-occipital regions with transcranial magnetic stimulation, bidirectionality was interrupted (Rothen et al. 2010). Specifically, a conditioned response was evident for colour patches, but no conditioned response occurred for graphemes. These results indicate that the same brain areas that have been shown to be involved in unidirectional synaesthetic colour experiences (Estermann, Verstynen, Ivry, and Robertson 2006; Muggleton, Tsakanikos, Walsh, and Ward 2007) are also involved in implicit bidirectionality.

What becomes clear from these empirical findings is that, in many cases, synaesthesia is triggered conceptually rather than by the physical presence of the inducer. Thus, synaesthesia depends on the interpretation of the inducers and their meaning rather than the mere appearance of particular stimuli. Rather than being caused by low-level pre-wired connections between sensory representations, higher-order representations in the brain seem to be responsible for the particular synaesthetic experiences. As a consequence, a representation of the inducer-concurrent relationship must be stored in the brain and this representation is activated in the absence of physical stimulation.

From these considerations, it seems obvious that synaesthesia is represented in the semantic knowledge system. However, the question how synaesthetic concurrents are represented in the semantic knowledge structure has not received much attention yet. Here, I propose that synaesthetic concurrents are represented as additional features in the semantic network, and, via spreading activation, these features allow for supplementary 
connections (cf. Collins and Loftus 1975; Cree and McCrae 2003). That is, associations may be easily formed in synaesthetes which are not obvious for non-synaesthetes and these associations may be expressed as creative thoughts, enriched memory representations, and in general a richer world of experience.

For example, consider the case of a synaesthete who has colour experiences for letters and, in addition, words are also coloured either according to the dominant vowel or the first letter. Specifically, the letter A may trigger the colour red, and similarly words such as tailor, mail, or acid may also trigger the colour red. Suppose that the colour red is represented in the semantic network with connections to other objects such as tomato, fire engine, and anger. For this particular person, associations between tailor and tomato, mail and fire engine, etc. may be more easily established than for a person without synaesthesia. Thus, via spreading activation, this person may generate easily curious thoughts and ideas that make the world of experiences richer compared to non-synaesthetes. In Figure 1, this example of an activated semantic network in response to the letter $\mathrm{A}$ for a synaesthete (left) and a non-synaesthete (right) is illustrated.

\section{Synaesthete}

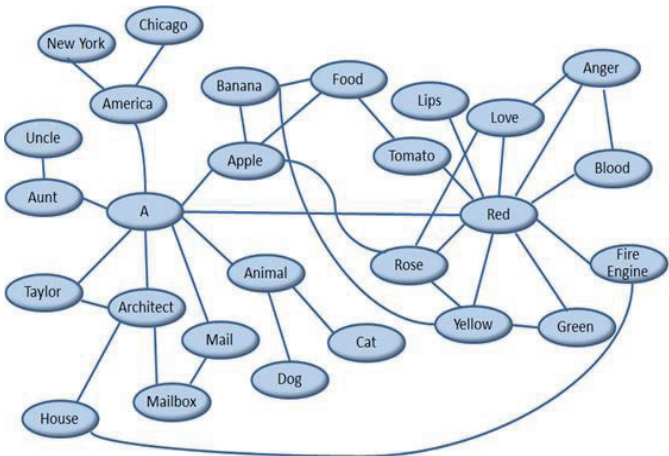

Non-Synaesthete

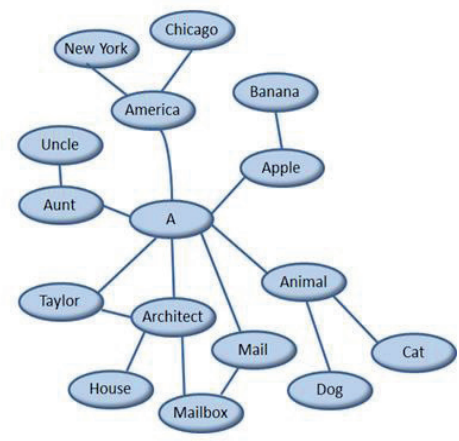

Figure 1. Illustration of exemplary activations in the semantic network in response to the letter $\mathrm{A}$ in a synaesthete with a corresponding red colour experience (left) and a non-synaesthetic control (right).

Having an enriched semantic network provides for richer encoding and for additional retrieval cues which may be the basis for a memory advantage. Moreover, the presence of additional connections between nodes in the semantic network may also be the basis for the increased creativity in synaesthesia. There is suggestive evidence for a link between synaesthesia 
and occupation with creative arts (cf. Rothen and Meier 2010b; Ward et al. 2008).

Inspired by the findings on the cognitive consequences of synaesthesia for memory, creativity, and imagery, and by findings from neuroimaging studies, we have recently investigated whether synaesthesia would be associated with a distinct cognitive style (Meier and Rothen 2013). Specifically, we have hypothesized that in grapheme-colour synaesthesia a preference for both verbal and visual cognitive styles may be prevalent. The results of two experiments confirmed this assumption. Moreover, our results suggested that a preference for a more vivid imagery style is also prevalent for other forms of synaesthesia, in particular those that involve colour (i.e., grapheme-colour and sound-colour), and in the case of the absence of grapheme-colour synaesthesia, those that involved multiple other forms of synaesthesia such as lexical-gustatory and sequence-space. Thus, in the absence of colour synaesthesia, the presence of multiple forms of synaesthesia also seemed to induce a richer world of experience that was then sufficient to enhance the preference for a vivid imagery cognitive style.

As noted, for grapheme-colour synaesthesia, both a verbal and a vivid imagery style co-occurred. Graphemes are instances of sequences and thus, they involve serial, analytic processing, favouring a verbal cognitive style. In contrast, colours are visual instances, which involve parallel or holistic processing, thus favouring a visual cognitive style. The combination of a verbal and a vivid imagery visual style in grapheme-colour synaesthetes and their propensity to switch easily between these styles may, in fact, be at the core of the cognitive benefits related to grapheme-colour synaesthesia.

To conclude, I would like to draw attention again to the issue of univs. bi-directionality. One may wonder why synaesthesia should be a unidirectional phenomenon at all. If letters induce colour experiences, then why do colours not also induce letter experiences? The properties of the semantic representation system, in particular the fan-effect, may provide a clue to answer this question (cf. Rothen et al. 2010). The term 'fan' refers to the number of facts associated with a particular concept. Anderson (1974) showed that recognition latencies increased as the concepts were associated with more features. In the domain of synaesthesia, inducers are typically cultural artefacts, that is, stimuli such as numerals, letters and words which are comprehended only after extensive experience. In contrast, concurrents represent more natural categories which are "just there" in the outside world and which can be comprehended without cultural education. If cultural artefacts (i.e., inducers) are more symbolic and less concrete than natural categories (i.e., concurrents), then an inducer would activate fewer nodes 
compared to a concurrent (cf. Collins and Loftus 1975). By this explanation, the association between inducer and concurrent is stronger and therefore the threshold for a conscious experience may be crossed more quickly. Reciprocally, the association between concurrent and inducer is weaker and therefore the threshold for a conscious experience may not be crossed. However, using appropriate measures, its existence can be demonstrated nevertheless as shown in studies on implicit bidirectionality.

I propose that future research should focus more on the semantic representation of synaesthesia. As suggested recently, in general, synaesthesia may be typically a semantic phenomenon and thus, low-level synaesthesia may rather be the exception than the rule (e.g., Mroczko-Wasowicz and Werning 2012; Nikolic et al. 2011; Rothen et al. 2013). However, higher level representation may be a prerequisite for cognitive consequences of synaesthesia in domains such as memory and creativity, and may determine the preferred cognitive processing style.

\section{References}

Anderson, John R. 1974. "Retrieval of propositional information from long-term memory." Cognitive Psychology 6: 451-474.

Baron-Cohen, Simon and John E. Harrison. 1997. Synaesthesia: Classic and Contemporary Readings. Cambridge: Blackwell.

Baron-Cohen, Simon, Maria A. Wyke, and Colin Binnie. 1987. "Hearing words and seeing colours: an experimental investigation of a case of synaesthesia." Perception 16: 761-767.

Barnett, Kylie J., John J. Foxe, Sophie Molholm, Simon P. Kelly, Shani Shalgi, Kevin J. Mitchell and Fiona N. Newell. 2008. "Differences in early sensoryperceptual processing in synesthesia: A visual evoked potential study." NeuroImage, 43, 605-613.

Brugger, Peter, Daria Knoch, Christine Mohr and Lorena R.R. Gianotti. 2004. "Is digit-color synaesthesia strictly unidirectional? Preliminary evidence for an implicitly colored number space in three synaesthetes." Acta Neuropsychologica 2(3): 252-258.

Cohen Kadosh, Roi and Avishai Henik. 2006. "Color congruity effect: where do colors and numbers interact in synesthesia?" Cortex 59: 259-263.

Collins, Allan M., and Elizabeth F. Loftus. 1975. "A spreading-activation theory of semantic processing." Psychological Review 82: 407-428.

Cree, George S., and Ken McRae. 2003. "Analyzing the factors underlying the structure and computation of the meaning of chipmunk, cherry, chisel, cheese, 
and cello (and many other such concrete nouns)." Journal of Experimental Psychology: General 132: 163-201.

Day, Sean A. 2013. "Synesthesia: demographic aspects of synesthesia." www. daysyn.com/types-of-syn.html DOA: 20 Nov 2013.

Dixon, Mike J., and Daniel Smilek, Cera Cudahy and Philip M. Merikle. 2000. "Five plus two equals yellow." Nature 406: 365 .

Dixon, Mike J., Daniel Smilek, Patricia L. Duffy, Mark P. Zanna and Philip M. Merikle. 2006. "The role of meaning in grapheme-colour synaesthesia." Cortex 42: 243-252.

Edquist, Jessica, Anina N. Rich. Cobie Brinkman and Jason B. Mattingley. 2006. "Do synaesthetic colours act as unique features in visual search?" Cortex 42: 222-231.

Esterman, Michael, Timothy Verstynen, Richard B. Irvy and Lynn C. Robertson. 2006. "Coming unbound: disrupting automatic integration of synesthetic color and graphemes by transcranial magnetic stimulation of the right parietal lobe." Journal of Cognitive Neuroscience 18: 1570-1576.

Frith, Chris D. and Eraldo Paulesu. 1997. The physiological basis of synaesthesia. In Simon Baron-Cohen and John E. Harrison (eds.), Synaesthesia. Classic and Contemporary Readings, 123-137. Oxford, UK: Blackwell.

Grossenbacher, Peter G. and Christopher T. Lovelace. 2001. "Mechanisms of synesthesia: cognitive and physiological constraints." Trends in Cognitive Sciences 5.1: 36-41.

Hänggi, Jürgen, Diana Wotruba and Lutz Jäncke. 2011. “Globally altered structural brain network topology in grapheme-color synesthesia." The Journal of Neuroscience 31: 5816-5828.

Hubbard, Edward M., A. Cyrus Arman, Vilayanur S. Ramachandran and Geoffrey M. Boynton. 2005. "Individual differences among grapheme-color synesthetes: Brain-behavior correlations." Neuron 45: 975-985.

Hubbard, Edward M. and Vilayanur S. Ramachandran. 2011. "The cross-activation theory at 10." Journal of Neuropsychology 5: 152-177.

Johnson, Addie, Marieke Jepma and Ritske de Jong. 2007. "Colours sometimes count: Awareness and bi-directionality in grapheme-colour synaesthesia." The Quarterly Journal of Experimental Psychology 60: 1406-1422.

Knoch, Daria, Lorena R.R. Gianotti, Christine Mohr and Peter Brugger. 2005. "Synesthesia: when colors count." Cognitive Brain Research 25: 372-374.

Laeng, Bruno, Frode Svartdal and Hella Oelmann. 2004. "Does color synesthesia pose a paradox for early-selection theories of attention?" Psychological Science 15(4): 277-281.

Mattingley, Jason B., Anina N. Rich, Greg Yelland and John L. Bradshaw 2001. "Unconscious priming eliminates automatic binding of colour and alphanumeric form in synaesthesia." Nature 410; 29 March: 580-582.

Meier, Beat and Nicolas Rothen. 2007. "When conditioned responses 'fire back': Bidirectional cross-activation creates learning opportunities in synesthesia." Neuroscience 147: 569-572. 
Meier, Beat and Nicolas Rothen. 2013. "Grapheme-colour synaesthesia is associated with a distinct cognitive style." Frontiers in Psychology, 4: 632. doi: 10.3389/ fpsyg.2013.00632

Meier, Beat and Nicolas Rothen. 2013. Synaesthesia and memory. In Julia Simner and Edward M. Hubbard (eds.). Oxford Handbook of Synaesthesia, 692-706. Oxford University Press, Oxford, UK.

Mroczko-Wasowicz, Aleksandra and Markus Werning. 2012. "Synesthesia, sensory-motor contingency, and semantic emulation: how swimming stylecolor synesthesia challenges the traditional view of synesthesia." Frontiers in Psychology 3: 279. doi: 10.3389/fpsyg.2012.00279

Muggleton, Neil, Elias Tsakanikos, Vincent Walsh and Jamie Ward. 2007. "Disruption of synaesthesia following TMS of the right posterior parietal cortex." Neuropsychologia 45: 1582-1585.

Myles, Kathleen M., Mike J. Dixon, Daniel Smilek and Philip M. Merikle. 2003. "Seeing double: The role of meaning in alphanumeric-colour synaesthesia." Brain and Cognition 53: 342-345.

Nikolič, Danko, Uta M. Jürgens, Nicolas Rothen, Beat Meier and Aleksandra Mroczko. 2011. "Swimming-style synesthesia." Cortex 47: 874-879.

Novich, Scott, Sherry Cheng and David M. Eagleman. 2011. "Is synaesthesia one condition or many? A large-scale analysis reveals subgroups." Journal of Neuropsychology 5: 353-371.

Palmeri, Thomas J., Randolph Blake, René Marois, Marci A. Flanery and William Whetsell, Jr. 2002. "The perceptual reality of synesthetic colors." Proceedings of the National Academy of Science 99; March 19: 4127-4131.

Radvansky, Gabriel A., Bradley S. Gibson and Windy M. McNerney. 2011. "Synesthesia and memory: Color congruency, von Restorff, and false memory effects." Journal of Experimental Psychology: Learning, Memory, and Cognition 37: 219-229.

Ramachandran, Vilayanur S. and Edward M. Hubbard. 2001a. "Psychophysical Investigations into the Neural Basis of Synaesthesia." Proceedings of the Royal Society of London, B 268: 979-983.

Ramachandran, Vilayanur S. and Edward M. Hubbard. 2001b. "Synaesthesia A window into perception, thought and language." Journal of Consciousness Studies 8: 3-34.

Rothen, Nicolas and Beat Meier. 2009. "Do synesthetes have a general advantage in visual search and episodic memory? A case for group studies." PLOS ONE 4: e5037.

Rothen, Nicolas and Beat Meier. 2010a. "Grapheme-colour synaesthesia yields an ordinary rather than extraordinary memory advantage: Evidence from a group study." Memory 18: 258-264.

Rothen, Nicolas and Beat Meier. 2010b. "Higher prevalence of synaesthesia in art students." Perception 5: 718-720. 
Rothen, Nicolas, Beat Meier and Jamie Ward. 2012. "Enhanced memory ability: Insights from synaesthesia." Neuroscience \& Biobehavioral Reviews 36: 1952-1963.

Rothen, Nicolas, Danko Nikolič, Uta M. Jürgens, Aleksandra Mroczko-Wasowicz, Josephine Cock and Beat Meier. 2013. "Psychophysiological evidence for the genuineness of swimming-style colour synaesthesia." Consciousness and Cognition 22: 35-46.

Rothen, Nicolas, Thomas Nyffeler, Roman von Wartburg, René Martin Müri and Beat Meier. (2010). "Parieto-occipital suppression eliminates implicit bidirectionality in grapheme-colour synaesthesia." Neuropsychologia 48: 3482-3487.

Rouw, Romke and H. Steven Scholte. 2007. "Increased structural connectivity in grapheme-color synesthesia." Nature Neuroscience 10.6, June: 792-797.

Rouw, Romke and H. Steven Scholte. 2010. "Neural basis of individual differences in synesthetic experiences." Journal of Neuroscience 30: 6205-6213.

Sagiv, Noam, Jeffrey Heer and Lynn Robertson. 2006. "Does binding of synesthetic color to the evoking grapheme require attention?" Cortex 42: 232-242.

Simner, Julia, Catherine Mulvenna, Noam Sagiv, Elias Tsakanikos, Sarah A. Witherby, Christine Fraser, Kirsten Scott and Jamie Ward. 2006. "Synaesthesia: The prevalence of atypical cross-modal experiences." Perception 35: 1024-1033.

Ward, Jamie, Clare Jonas, Zoltan Dienes and Anil Seth. 2009. "Grapheme-colour synaesthesia improves detection of embedded shapes, but without preattentive 'pop-out' of synaesthetic colour." Proceedings of the Royal Society B-Biological Sciences 277: 1021-1026.

Ward, Jamie, Daisy Thompson-Lake, Roxanne Ely and Flora Kaminski. 2008. "Synaesthesia, creativity and art: What is the link?" British Journal of Psychology 99: 127-141.

Weiss, Peter H., and Gereon. R. Fink. 2009. "Grapheme-colour synaesthetes show increased grey matter volumes of parietal and fusiform cortex." Brain 132: 65-70.

Weiss, Peter H., Andreas Kalckert and Gereon. R. Fink. 2009. "Priming letters by colors: Evidence for the bidirectionality of grapheme-color synesthesia." Journal of Cognitive Neuroscience 21: 2019-2026.

Yaro, Caroline, and Jamie Ward. 2007. "Searching for Shereshevskii: What is superior about the memory of synaesthetes?" The Quarterly Journal of Experimental Psychology 60: 681-695. 